\title{
Angelman syndrome: advancing the research frontier of neurodevelopmental disorders
}

\author{
Benjamin D. Philpot • Coral E. Thompson • \\ Lisa Franco $\cdot$ Charles A. Williams
}

Received: 12 September 2010 / Accepted: 8 November 2010 /Published online: 3 December 2010

(C) Springer Science+Business Media, LLC 2010

\begin{abstract}
This report is a meeting summary of the 2010 Angelman Syndrome Foundation's scientific symposium on the neuroscience of UBE3A. Angelman syndrome is characterized by loss of speech, severe developmental delay, seizures, and ataxia. These core symptoms are caused by maternal allele disruptions of a single geneUBE3A. UBE3A encodes an E3 ubiquitin ligase that targets certain proteins for proteasomal degradation. This biology has led to the expectation that the identification of Ube3a protein targets will lead to therapies for Angelman syndrome. The recent discovery of Ube3a substrates such as Arc (activity-regulated cytoskeletal protein) provides new insight into the mechanisms underlying the synaptic function and plasticity deficits caused by the loss of Ube3a. In addition to identifying Ube3a substrates, there have also been recent advances in understanding UBE3A's integrated role in the neuronal repertoire of genes and protein
\end{abstract}

\section{B. D. Philpot}

Department of Cell and Molecular Physiology, Neuroscience Center, and Carolina Institute for Developmental Disabilities, University of North Carolina,

Chapel Hill, NC, USA

B. D. Philpot • C. E. Thompson • L. Franco • C. A. Williams Scientific Advisory Committee, Angelman Treatment and Research Institute, Angelman Syndrome Foundation, Aurora, IL, USA

\section{A. Williams}

Raymond C. Philips Unit, Division of Genetics and Metabolism, Department of Pediatrics,

University of Florida College of Medicine,

Gainesville, FL, USA

C. A. Williams $(\bowtie)$

P.O. Box 100296, HSC, Gainesville, FL 32610, USA

e-mail: willicx@peds.ufl.edu interactions. A developmental picture is now emerging whereby UBE3A gene dosage on chromosome 15 alters synaptic function, with deficiencies leading to Angelman syndrome and overexpression associated with classic autism symptomatology.

Keywords UBE3A · Angelman syndrome · Synapse · Autism $\cdot$ Ubiquitin $\cdot$ Activity-regulated genes

\section{Introduction}

The 2010 scientific symposium was held June $15-16$ in Chapel Hill, NC. The symposium is conducted annually by the Angelman Treatment and Research Institute, a part of the Angelman Syndrome Foundation (www.angelman.org). The theme for this year's symposium was "The Neuroscience of UBE3A from Genes to Behavior." The program consisted of invited speakers and other presentations and was attended by approximately 80 scientists. It became clear during the conference that Angelman syndrome research is providing a new vista into the understanding of neurodevelopmental disorders. Here, we first review some basic aspects of Angelman syndrome (AS) and then thematically summarize the meeting presentations.

In normal neurons, UBE3A is transcriptionally inactivated (imprinted) on the paternally derived allele of chromosome 15 and is active only on the maternally derived allele. All other somatic cells have biallelic transcription. In 1997, loss of Ube3a function was identified as the cause of AS (Kishino et al. 1997; Matsuura et al. 1997). The syndrome can occur by four different mutational mechanisms affecting the maternally derived chromosome 15: intragenic mutation, chromosome microdeletion of the gene, paternal uniparental disomy (absence 
of maternal chromosome 15), and a defect in the imprinting center that controls UBE3A transcription. The syndrome occurs in 1:12-15,000 births and is characterized by severe to profound cognitive delay, seizures, severely limited speech, pro-social behaviors, tremulous limb movements, and an ataxic gait (Williams et al. 2010).

\section{UBE3A targets and protein associations}

To understand the role of Ube $3 \mathrm{a}$ and potential therapies for AS, an important goal is to identify both the substrates that Ube3a targets for degradation and other Ube3a-interacting proteins. Moreover, the role of Ube3a must also be considered in the context of how it and its substrates might be regulated by neural activity. With this in mind, Michael E. Greenberg, the symposium's keynote speaker, provided an overview of how neuronal stimulation induces the transcription of hundreds of genes, including transcription factors and genes encoding synaptic proteins, as reviewed in Greer and Greenberg (2008). An increase in intracellular calcium appears to be the first step in this gene induction process. Calcium influx, typically through L-type voltagesensitive calcium channels and NMDA receptors, can initiate synapse-to-nucleus signaling. Calcium-mediated responses activate cytoplasmic proteins, including protein kinases, which translocate to the nucleus to phosphorylate and activate transcription factors. The cAMP-response elementbinding protein (CREB) was one of the first studied proteins involved in this type of induction. CREB acts on genes with CRE-response elements through a repressor complex spanning the promoter and upstream elements of these genes. Calcium-mediated phosphorylation of CREB increases its association with CREB binding protein and other proteins in the basal transcription complex to promote gene transcription. This intricate process involves changes in histone acetylation and recruitment of the RNAII polymerase complex to promote gene transcription.

The Greenberg lab has suggested that UBE3A is itself an activity-regulated gene (Flavell et al. 2008; Greer et al. 2010) and thus protein substrates of Ube3a would likely have similar activity-dependent expression. To identify new UBE3A targets, the Greenberg lab has taken advantage of a transgenic mouse with a hemagglutinin epitope-tagged version of ubiquitin (HA-ubiquitin) knocked into the HPRT locus. By crossing these mice with either wild-type or Ube3a-deficient mice, and comparing ubiquitination differences between the two crosses, the investigators were able to identify direct brain lysate proteins in AS mice that had reduced HA-ubiquitin tagging. Using this approach, they identified Sacsin as an Ube3a substrate (mutation in the Sacsin gene causes autosomal recessive spastic ataxia of Charlevoix-Saguenay). Then, by comparing sequences between Sacsin and another known UBE3A substrate, HHR23A, they identified a UBE3A-binding domain having loose sequence homology. Using this domain, they identified Arc (activity-regulated cytoskeletal protein) as a new UBE3A target (Greer et al. 2010). Arc is known to regulate AMPA receptor (AMPAR) trafficking and internalization at the synapse (Bramham et al. 2010). Accordingly, they found diminished surface counts of synaptic AMPAR receptors using array tomography (a technique in which ultra-thin sections of brain tissue are stained, imaged, and synapses visualized as a 3D reconstruction) and increased Arc protein levels in direct brain lysate analysis of AS mouse neurons (which lack Ube3a). The Greenberg lab hypothesized that the absence of Ube3a could lead to accumulations of Arc, which in turn could lead to increased AMPAR internalization. Notably, the converse could occur in situations of UBE3A excess, as in the $1 \%$ of individuals with autism arising from maternal duplications of the 15q11-q13 region (Christian et al. 2008; Cook et al. 1997), which spans the UBE3A gene. Increased UBE3A gene expression has been demonstrated for these duplications, both the interstitial ones and the isodicentric marker chromosomes (Herzing et al. 2002; Baron et al. 2006). Interestingly, Arc is known to be translationally inhibited by the Fragile X protein (FMRP), and the simplistic picture emerges that dysregulation of Arc protein homeostasis may be a commonality between AS and Fragile $\mathrm{X}$ mental retardation. It is not clear at this time whether inhibitors of the metabotropic glutamate 5 receptor, a therapeutic strategy being pursued in Fragile X, could be of benefit in AS.

Dr. Greenberg also reported on discovery of a new Ube3a substrate involved with the EphB subfamily of receptor tyrosine kinases. Eph receptors are known to be enriched at synapses and are important in regulating dendritic spine density. The EphB receptors interact with ephrin ligands and regulate dendritic development through small GTPases of the Rho family (Rho, Rac, and Cdc42) by activation of guanine nucleotide exchange factors (GEFs) (Murai and Pasquale 2003). GEFs serve as a switch to convert GTPases from an inactive GDP to an active GTP state. The GTP-bound forms can interact with downstream effectors that control cytoskeletal rearrangements. EphBlinked Rho activation appears to inhibit dendritic spine outgrowth, while Rac activation antagonizes this effect and promotes outgrowth. Proper dendritic spine formation may require a balance between actions of these GEFs. The Greenberg lab determined that a newly identified Ube3a substrate, named Ephexin 5, acts as a GEF for Rho (Margolis et al. 2010). Consistent with Ephexin5 being a Ube3a substrate, Ube3a-deficient neurons exhibit increased Ephexin 5 levels. This raises the possibility that loss of UBE3A function could lead to restricted synaptic development; conversely, duplication of UBE3A (as seen in some 
cases of autism) could result in diminished Ephexin 5 levels with promotion of spine development.

Other labs are also using new, sophisticated technologies to identify Ube3a substrates. Peter Howley, a long-time investigator into the role of Ube3a, reported on an unbiased proteomics approach to identify substrates and regulators of Ube3a. With most proteomic studies, it is difficult to determine the physiologically relevant proteins among the large number of nonspecific interacting proteins dominating a mass spectral analysis. To overcome this problem, the Howley lab is collaborating with Wade Harper's lab to improve the identification of relevant interacting proteins and the analysis of interaction networks by using a newly established platform (CompPASS). CompPASS uses catalytically inactive forms of the three major isoforms of UBE3A to identify high confidence interacting proteins (Sowa et al. 2009). Initial work with this approach used human embryonic kidney cells but is now being extended into human neural cells using a TET-regulatable human neuroblastoma SH-SY5Y cell line to identify neural-specific substrates. The expectation is that this new approach will soon lead to the identification of novel Ube3a substrates, providing additional therapeutic avenues in AS.

Lawrence T. Reiter reported on his work toward identifying substrates and interacting proteins for the Drosophila ortholog of UBE3A (dUBE3A). Dr. Reiter used proteomic profiling in Drosophila heads to show that dUBE3A may regulate Punch (GTP cyclohydrolase I, GCH1). GCH1 catalyzes the first and rate-limiting step in tetrahydrobiopterin biosynthesis and thus is a key regulator of monoamine (dopamine/serotonin) synthesis in flies and humans. Consequently, dUBE3A overexpression elevates tetrahydrobiopterin levels while diminished expression of dUBE3A has the opposite effect on both GCH1 transcripts and dopamine levels. Dr. Reiter proposed that this regulatory effect could be due to a transcriptional co-activation function of dUBE3A since an enzymatically inactive form of dUBE3A (dUBE3A-C/A) is also able to elevate dopamine levels through GCH1. Performing rapid genetic and functional assays in Drosophila is an advantage of this model system, one that has already been used to demonstrate the importance of UBE3A in dendritic structure (Lu et al. 2009).

Using the more traditional, but still powerful, approach of yeast-two-hybrid screening and affinity proteomics, Konstantin Matentzoglu and his group identified the HECT protein HERC2 as a novel interaction partner of UBE3A. Interestingly, the HERC2 gene is located in close proximity to UBE3A and frequently found co-deleted with UBE3A in individuals with AS. HERC2 contains a HECT and three Rcc1-like domains (RLDs). The research shows that one of the RLDs, the Rcc1b domain of HERC2, not only mediates binding to Ube3a but also greatly enhances Ube3a ligase activity. Herc2 directly interacts with all three isoforms of
Ube3a. Herc2 recruits Ube3a into complexes and enhances auto-ubiquitination; furthermore, substrate ubiquitination of Ring $1 b$ appears to be activated in the same manner. The overlapping tissue distribution of Ube3a and Herc2 suggests a tantalizing possibility for functional interactions, although the physiological relevance of these potential interactions remains unknown.

\section{Effects of Ube3a loss on synaptic plasticity, neuron functions, and cell survival}

In addition to the importance of identifying substrates and interacting partners for Ube3a, another important approach for guiding therapeutic approaches is to understand the neurological consequences of reduced Ube 3 a expression. Towards this end, Ben Philpot has used a mouse model of AS to demonstrate that UBE3A is required for neocortical plasticity in vitro as well as for experience-driven plasticity in vivo (Yashiro et al. 2009). His work and that of others demonstrate deficits in AS mouse for long-term potentiation and long-term depression, indicating that the loss of Ube3a leads to a severe synaptic rigidity that likely underlies impaired learning in individuals with AS. New data show that reduced Ube3a causes a loss of synaptic connectivity across a variety of neuronal cell types and that this deficit in connectivity may be one of several factors limiting experience-driven synaptic learning in vivo. The Philpot lab has previously shown that excitatory synapse development is blunted in Ube3a-deficient mice by postnatal day 21. The Philpot lab now finds that there are also severe deficits in inhibitory synapse development, but these deficits arise later in development. The deficits in inhibitory transmission appear to arise from decreased synaptic contacts made by inhibitory interneurons onto excitatory pyramidal neurons. It is unclear at this time whether the change in inhibition is primary or adaptive. However, he speculated that the loss of inhibition is maladaptive, as the mice have increased seizure susceptibility that is likely to arise from a severe loss of inhibition. These observations suggest that an imbalance between excitation and inhibition in the neocortex may contribute to epilepsy and neocortical dysfunction associated with AS.

What might underlie the synaptic and plasticity deficits observed in Ube3a-deficient mice? Although many factors are likely to contribute, the increases in Arc expression observed in Ube3a-deficient mice (noted above) could explain both the loss of functional synapses, due to the loss of AMPARs, and deficits in synaptic plasticity, due to aberrant AMPAR trafficking. Indeed, it is now clear that Arc expression must be tightly regulated for normal experience-driven plasticity in learning. Along these lines, Jason D. Shepherd reported on recent data demonstrating 
that genetic deletion of Arc prevents experience-dependent plasticity in the developing visual cortex (McCurry et al. 2010). Arc knockout mice exhibited deficits in ocular dominance plasticity due to deficient depression of the deprived eye responses, similar to that observed in AS model mice (Yashiro et al. 2009; Sato and Stryker 2010). Arc knockout mice also exhibit deficits in stimulus-specific response potentiation (SRP), a relatively newly identified form of experience-dependent plasticity (Frenkel et al. 2006). In SRP, daily exposure to a horizontal grating stimulus of a single orientation selectively potentiates the amplitude of visual evoked potentials to that orientation, but not other orientations. These deficits occurred despite normal visual acuity and retinotopic organization, also similar to what has been observed in the AS mouse model. Thus, accumulating data suggest that having either too little or too much Ube3a, and by extension Arc, causes a severe impairment in synaptic plasticity.

In addition to plasticity defects, the loss of Ube3a can alter a number of other neuronal properties. Indeed, Hanoch Kaphzan reported that the loss of Ube3a alters intrinsic membrane properties in hippocampal area CA1 neurons. These changes may be caused, at least in part, by increased expression of the Na/K-ATPase alpha1 subunit, Ankyrin-B and Ankyrin-G, all three known to interact with the ATPase transporter. The $\mathrm{Na} / \mathrm{K}$-ATPase transporter consists of alpha and beta subunits, and at least four genes encode for different isoforms of the alpha subunits. Missense mutations in the alpha 2 gene (ATP1A2) have been associated with familial hemiplegic migraine, a disorder in which some affected individuals can also have seizures and ataxia. Because $\mathrm{Na} / \mathrm{K}$-ATPases play a seminal role in controlling neuronal excitability, it is tempting to speculate that the observed abnormalities could be related to the seizure liability observed in individuals with AS.

Simone Küehnle used established non-neural mammalian cell lines and RNAi-mediated downregulation of Ube3a to demonstrate that reduced Ube3a levels result in severe cytotoxicity. He also found that ectopic Ube3a expression is cytotoxic too, indicating that Ube3a levels have to be delicately balanced in mammalian cells, contributing once again to the recurring theme that either the loss or overexpression of Ube3a can lead to many similar defects. He then performed studies showing that, for cells depleted for Ube3a, CaMKII overexpression can partially rescue the cytotoxic phenotype. CaMKII activity is known to be perturbed in AS mouse neurons (Weeber et al. 2003). Hence, the cytotoxicity of Ube3a depletion, as observed in non-neuronal cell lines, may at least in part involve the same pathways that are deregulated in the AS mouse model. Dr. Küehnle's research with established cell lines may be of potential use to elucidate the pathways involved in AS development.
New mouse models of Angelman syndrome and Ube3a imprinting

Future advances in AS will rely heavily on the development of new mouse models and their creative use. Scott Dindot has developed an Ube3a-YFP reporter mouse (Dindot et al. 2008) and is now using crosses of this mouse having the YFP reporter on either the maternal or paternal allele to examine the allelic expression patterns of Ube3a during neurogenesis. Current observations suggest that both paternal and maternal alleles express Ube3a in the stem cell niches lining the lateral ventricles and in the dentate gyrus of the hippocampal formation. Progenitor cells and immature neurons both express Ube3a, while expression of Ube3a in mature neurons is derived primarily from the maternal allele. Notably, low levels of expression were detected from the paternal allele, demonstrating that the paternal allele remains functional and intact. Collectively, these data suggest that imprinting of Ube $3 \mathrm{a}$ is specific to neuronal cells, as previously reported (Yamasaki et al. 2003), but is not complete until the later stages of neuronal maturation.

While most mouse models of AS have involved selective deletion of Ube3a (Jiang et al. 1998; Miura et al. 2002), $70 \%$ of individuals with AS have a much larger deletion from $15 \mathrm{q} 11$ to q13. To create a more realistic model of the most common form of AS, Yong-Hui Jiang and his coworkers generated mutant mice with a $1.6 \mathrm{Mb}$ chromosomal deletion that spans Ube3a to Gabrb3, including deletion of Atp10a. Homozygous deletion mice show cleft palate and die in the perinatal period, but the heterozygotes survive and show parent-of-origin phenotypes (Jiang et al. 2010). Expression analysis of maternal and paternal deletion mice confirmed that the Ube3a gene is exclusively maternally expressed in brain and that the Atp10a and Gabrb3 genes are biallelically expressed in all brain subregions studied. The maternal deletion mice showed many of the behavioral features seen in other Ube3a-deficient mouse models. In addition, ultrasonic vocalization recordings in newborns revealed that maternal deletion pups emitted significantly more vocalizations than wild-type littermates, but the behavioral correlate to communication deficits observed in human AS is unclear. This mouse model will be invaluable to future AS research and will help to provide a biological basis for the more severe deficits in speech and epilepsy observed in AS individuals with $15 \mathrm{q} 11-\mathrm{q} 13$ deletions compared to those with Ube3a mutations (Lossie et al. 2001).

\section{Induced pluripotent stem cells, gene transcription, and vector strategies}

New technologies in biomedical research show promise in advancing our understanding on AS. Marc Lalande ele- 
gantly described how he and Stormy Chamberlain are using induced pluripotent stem (iPS) cells to develop an innovative approach to study AS. Their lab has established two human AS deletion and two normal control iPS cell lines derived from fibroblasts (Chamberlain et al. 2010). The iPS cell lines express NANOG and OCT4, two markers that characterize the undifferentiated stem cell state. The iPS cell lines can be differentiated into mature neurons, using a technique that mimics neural development in the embryo. During this process of in vitro neurogenesis, there is upregulation of PAX6, a marker of neuronal precursors, at early stages, followed by expression of MAP2, Synapsin I and III tubulin in neurons. Single-cell recordings from the iPS-derived neurons demonstrate that these cells fire action potentials and exhibit spontaneous synaptic activity, hallmarks of mature neurons. A marked decrease in Ube3a mRNA and protein is observed in neurons derived from AS iPS cells, indicating that silencing of the paternal UBE3A allele occurs in this in vitro platform, similar to what is likely to occur in vivo. AS neurons derived from iPS cell lines may thus provide important insights about the developmental timing and regulation of UBE3A imprinting and for characterizing the functional abnormalities in human AS neurons.

The imprinting of Ube3a presents unique treatment opportunities. One strategy for developing an AS therapeutic is to activate the silenced, but intact, paternal Ube3a allele. Towards this end, Barbara Bailus presented an update on work with David Segal in developing artificial transcription factors (ATFs) that may serve such a purpose. By attaching an activation or repression domain to a zinc finger construct, an ATF is formed that can enhance or suppress transcriptional activity. Two approaches are being explored to activate the paternal copy of murine Ube3a in mouse cells. The direct approach targets an activator ATF to the promoter region of the paternal Ube3a, while the indirect approach will use repressor ATFs to silence the Ube3a antisense transcript thought to silence the paternal copy of Ube3a. While still preliminary, this approach represents a promising new front on the development of AS therapeutics.

Clearly, a number of approaches to develop AS therapeutics rely on methodologies that restore normal Ube3a levels in individuals with AS. To explore the possibility that this might be achievable with advances in gene therapy, Ron Mandel reviewed the use of recombinant adeno-associated viral vectors (rAAV) for adjusting gene expression in the brain. Transduction efficiency has steadily increased since the first use of the rAAV vectors in 1996. To date, rAAV2 has been used in five human clinical trials for neurological disorders. As rAAV2 production methods have improved, the purity of the vector and the amount recovered through the production process has increased. In addition, with the advent of alternative AAV capsid serotypes, vector titers have increased, and brain transduction has become more efficient. Nevertheless, there continue to be considerable problems in achieving global cellular transduction. Neurons that are transduced (glial cells do not become transduced) have generally robust and durable gene expression. The rAAV has a size constraint of approximately $5 \mathrm{~kb}$ of genomic code (including the promoter), the approximate size of the UBE3A cDNA, so packaging may be difficult. Also, gene copy number varies widely in transduced neurons, and this may be problematic for UBE3A since both diminished and increased cellular levels of Ube3a appear to be harmful. While gene therapy may not present a readily viable option for restoring Ube3a levels in the brain and treating AS, the expectation is that advances in gene therapy may someday make this approach viable.

\section{Human clinical and behavioral studies}

Mark Nespeca provided an overview of the clinical conundrums associated with epilepsy in AS. There is no salient hypothesis as to why seizures are so prevalent in the syndrome, and the clinical treatment with anticonvulsants remains largely empirical but fairly effective. To date, no class of anticonvulsants has shown a clearly superior benefit. Thus, an important future goal is to understand the pathophysiology underlying the severe and relatively intractable forms of epilepsy in AS so that more rationally based anticonvulsants can be designed.

Due to the relative rarity of AS, best treatment practices are often derived from extensive parent questionnaires. With this in mind, Sarika Peters provided an update of a web-based parent questionnaire project aimed at delineating the use of traditional and alternative treatments for challenging behaviors in AS. More than half $53 \%$ of 333) the caregivers reported individuals with AS using at least one alternative treatment. The most popular alternative treatments included melatonin, omega-3 fatty acids/fish oil, folic acid, and magnesium. However, the efficacy of these approaches still needs to be studied under rigorous control conditions, as was recently done by Peters et al. (2010) for analysis of folic acid and betaine as a putative AS therapeutic. In addition to using surveys to help determine best practices in AS, surveys have also helped to highlight the remarkable strain on caregivers. Frances Ulman presented results from an online anonymous questionnaire survey investigating parenting experiences in mothers of a child with a developmental handicap. A total of 211 mothers completed the questionnaire and, of the sample, 30 had a child with AS. Mothers of a child with AS demonstrated a unique profile of caregiving experiences, including devoting significantly greater time to caregiving 
and physical demands. Thus, there is an unmet need to develop therapies that can alleviate the behavioral issues surrounding Angelman syndrome, and great consideration needs to be given to the extreme challenges of being a caregiver.

A common misperception is that individuals with AS are incapable of learning. Jane Summers is helping to debunk this misperception. She reported on her efforts to develop a behavioral battery to assess memory, motor imitation, and motor performance skills in individuals with AS. She studied 12 children, ages 3.6 to 12.7 years, and used structured and reproducible assessment protocols. Her work demonstrates that there are a range of abilities among individuals with AS and some individuals exhibit relatively well-developed motor performance skills and an ability to reproduce actions from memory. Participants' skill levels were tested several times and remained stable over the assessment period. This work provides a way to evaluate key learning skills in individuals with AS and study the impact of different therapeutic approaches on their ability to learn.

More details of the findings from the conference are available in the abstracts at the Angelman Syndrome Foundation website (www.angelman.org).

\section{Conclusion}

We are gaining a better understanding of the biological actions of UBE3A as more neuroscientists investigate its role in neural plasticity and learning. Newly discovered UBE3A targets and interacting proteins are creating speculation about therapeutic, drugable targets, and this is an exciting change in the research landscape for AS. While deficiency of Ube3a in brain causes AS, overexpression appears to be associated with intellectual impairments and developmental abnormalities in the autism spectrum. Whether an increase in Ube3a dosing alone is the primary cause for the autism symptomatology is currently unknown. Continued research into the area of autism may thus bring better understanding about AS, and continued research on UBE3A's role in AS may likewise bring benefits to those with autism.

\section{References}

Baron CA, Tepper CG, Liu SY, Davis RR, Wang NJ, Schanen NC, et al. Genomic and functional profiling of duplicated chromosome 15 cell lines reveal regulatory alterations in UBE3Aassociated ubiquitin-proteasome pathway processes. Hum Mol Genet. 2006;15(6):853-69.

Bramham CR, Alme MN, Bittins M, Kuipers SD, Nair RR, Pai B, et al. The Arc of synaptic memory. Exp Brain Res. 2010;200 (2):125-40
Chamberlain SJ, Chen P-F, Ng KY, Bourgois-Rocha F, Lemtiri-Chlieh $\mathrm{F}$, Levine ES, et al. Induced pluripotent stem cell models of the genomic imprinting disorders, Angelman and Prader-Willi syndrome. PNAS, USA; 2010 (in press).

Christian SL, Brune CW, Sudi J, Kumar RA, Liu S, Karamohamed S, et al. Novel submicroscopic chromosomal abnormalities detected in autism spectrum disorder. Biol Psychiatry. 2008;63(12):1111-7.

Cook Jr EH, Lindgren V, Leventhal BL, Courchesne R, Lincoln A, Shulman C, et al. Autism or atypical autism in maternally but not paternally derived proximal $15 \mathrm{q}$ duplication. Am J Hum Genet. 1997;60(4):928-34.

Dindot SV, Antalffy BA, Bhattacharjee MB, Beaudet AL. The Angelman syndrome ubiquitin ligase localizes to the synapse and nucleus, and maternal deficiency results in abnormal dendritic spine morphology. Hum Mol Genet. 2008;17(1):111-8.

Flavell SW, Kim TK, Gray JM, Harmin DA, Hemberg M, Hong EJ, et al. Genome-wide analysis of MEF2 transcriptional program reveals synaptic target genes and neuronal activity-dependent polyadenylation site selection. Neuron. 2008;60(6):1022-38.

Frenkel MY, Sawtell NB, Diogo AC, Yoon B, Neve RL, Bear MF. Instructive effect of visual experience in mouse visual cortex. Neuron. 2006;51(3):339-49.

Greer PL, Greenberg ME. From synapse to nucleus: calciumdependent gene transcription in the control of synapse development and function. Neuron. 2008;59(6):846-60.

Greer PL, Hanayama R, Bloodgood BL, Mardinly AR, Lipton DM, Flavell SW, et al. The Angelman Syndrome protein Ube3A regulates synapse development by ubiquitinating arc. Cell. 2010;140(5):704-16.

Herzing LB, Cook Jr EH, Ledbetter DH. Allele-specific expression analysis by RNA-FISH demonstrates preferential maternal expression of UBE3A and imprint maintenance within 15q11q13 duplications. Hum Mol Genet. 2002;11(15):1707-18.

Jiang YH, Armstrong D, Albrecht U, Atkins CM, Noebels JL, Eichele $\mathrm{G}$, et al. Mutation of the Angelman ubiquitin ligase in mice causes increased cytoplasmic p53 and deficits of contextual learning and long-term potentiation [see comments]. Neuron. 1998;21(4):799-811.

Jiang YH, Pan Y, Zhu L, Landa L, Yoo J, Spencer C, et al. Altered ultrasonic vocalization and impaired learning and memory in Angelman syndrome mouse model with a large maternal deletion from Ube3a to Gabrb3. PLoS One 2010;5(8). doi:10.1371/ journal.pone. 0012278 .

Kishino T, Lalande M, Wagstaff J. UBE3A/E6-AP mutations cause Angelman syndrome [published erratum appears in Nat Genet 1997 Apr;15(4):411]. Nat Genet 1997;15(1):70-3.

Lossie AC, Whitney MM, Amidon D, Dong HJ, Chen P, Theriaque D, et al. Distinct phenotypes distinguish the molecular classes of Angelman syndrome. J Med Genet. 2001;38(12):834-45.

Lu Y, Wang F, Li Y, Ferris J, Lee JA, Gao FB. The Drosophila homologue of the Angelman syndrome ubiquitin ligase regulates the formation of terminal dendritic branches. Hum Mol Genet. 2009; 18(3):454-62.

Margolis SS, Salogiannis J, Lipton DM, Mandel-Brehm C, Wills ZP, Mardinly AR, et al. EphB-mediated degradation of the RhoA GEF Ephexin5 relieves a developmental brake on excitatory synapse formation. Cell. 2010;143(3):442-55.

Matsuura T, Sutcliffe JS, Fang P, Galjaard RJ, Jiang YH, Benton CS, et al. De novo truncating mutations in E6-AP ubiquitin-protein ligase gene (UBE3A) in Angelman syndrome. Nat Genet. 1997;15(1):74-7.

McCurry CL, Shepherd JD, Tropea D, Wang KH, Bear MF, Sur M. Loss of Arc renders the visual cortex impervious to the effects of sensory experience or deprivation. Nat Neurosci. 2010;13(4):450-7.

Miura K, Kishino T, Li E, Webber H, Dikkes P, Holmes GL, et al. Neurobehavioral and electroencephalographic abnormalities in 
Ube3a maternal-deficient mice. Neurobiol Dis. 2002;9(2):14959.

Murai KK, Pasquale EB. 'Eph'ective signaling: forward, reverse and crosstalk. J Cell Sci. 2003;116(Pt 14):2823-32.

Peters SU, Bird LM, Kimonis V, Glaze DG, Shinawi LM, Bichell TJ, et al. Double-blind therapeutic trial in Angelman syndrome using betaine and folic acid. Am J Med Genet A. 2010;152A(8):19942001.

Sato M, Stryker MP. Genomic imprinting of experience-dependent cortical plasticity by the ubiquitin ligase gene Ube3a. Proc Natl Acad Sci USA. 2010;107(12):5611-6.

Sowa ME, Bennett EJ, Gygi SP, Harper JW. Defining the human deubiquitinating enzyme interaction landscape. Cell. 2009;138 (2):389-403.
Weeber EJ, Jiang YH, Elgersma Y, Varga AW, Carrasquillo Y, Brown SE, et al. Derangements of hippocampal calcium/ calmodulin-dependent protein kinase II in a mouse model for Angelman mental retardation syndrome. J Neurosci. 2003;23 (7):2634-44

Williams CA, Driscoll DJ, Dagli AI. Clinical and genetic aspects of Angelman syndrome. Genet Med. 2010;12(7):385-95.

Yamasaki K, Joh K, Ohta T, Masuzaki H, Ishimaru T, Mukai T, et al. Neurons but not glial cells show reciprocal imprinting of sense and antisense transcripts of Ube3a. Hum Mol Genet. 2003;12 (8):837-47.

Yashiro K, Riday TT, Condon KH, Roberts AC, Bernardo DR, Prakash R, et al. Ube3a is required for experience-dependent maturation of the neocortex. Nat Neurosci. 2009;12(6):777-83. 